\title{
Resolving Ultrafast Heating of Dense Cryogenic Hydrogen
}

\author{
U. Zastrau, ${ }^{1,2,}{ }^{*}$ P. Sperling, ${ }^{3}$ M. Harmand ${ }^{4}$ A. Becker, ${ }^{3}$ T. Bornath, ${ }^{3}$ R. Bredow, ${ }^{3}$ S. Dziarzhytski, ${ }^{4}$ T. Fennel, ${ }^{3}$ \\ L. B. Fletcher, ${ }^{2}$ E. Förster, ${ }^{1,5}$ S. Göde,${ }^{3}$ G. Gregori,${ }^{6}$ V. Hilbert, ${ }^{1}$ D. Hochhaus, ${ }^{7}$ B. Holst,${ }^{3}$ T. Laarmann, ${ }^{4,8}$ \\ H. J. Lee, ${ }^{2}$ T. Ma, ${ }^{9}$ J. P. Mithen, ${ }^{6}$ R. Mitzner, ${ }^{10}$ C. D. Murphy, ${ }^{6}$ M. Nakatsutsumi, ${ }^{11}$ P. Neumayer, ${ }^{7}$ A. Przystawik, ${ }^{4}$ \\ S. Roling, ${ }^{10}$ M. Schulz, ${ }^{4}$ B. Siemer, ${ }^{10}$ S. Skruszewicz, ${ }^{3}$ J. Tiggesbäumker, ${ }^{3}$ S. Toleikis, ${ }^{4}$ T. Tschentscher,${ }^{11}$ \\ T. White, ${ }^{6}$ M. Wöstmann, ${ }^{10}$ H. Zacharias, ${ }^{10}$ T. Döppner, ${ }^{9}$ S. H. Glenzer, ${ }^{2}$ and R. Redmer ${ }^{3}$ \\ ${ }^{1}$ Institut für Optik und Quantenelektronik, Friedrich-Schiller-Universität, Max-Wien-Platz 1, 07743 Jena, Germany \\ ${ }^{2}$ SLAC National Accelerator Laboratory, 2575 Sand Hill Road, Menlo Park, California 94025, USA \\ ${ }^{3}$ Institut für Physik, Universität Rostock, D-18051 Rostock, Germany \\ ${ }^{4}$ Deutsches Elektronen-Synchrotron DESY, Notkestrasse 85, D-22607 Hamburg, Germany \\ ${ }^{5}$ Helmholtz-Institut Jena, Fröbelstieg 3, 07743 Jena, Germany \\ ${ }^{6}$ Department of Physics, Clarendon Laboratory, University of Oxford, Parks Road, Oxford OX1 3PU, United Kingdom \\ ${ }^{7}$ Extreme Matter Institute, GSI Helmholtzzentrum für Schwerionenforschung, 64291 Darmstadt, Germany \\ ${ }^{8}$ The Hamburg Centre for Ultrafast Imaging CUI, 22761 Hamburg, Germany \\ ${ }^{9}$ Lawrence Livermore National Laboratory, 7000 East Avenue, Livermore, California 94550, USA \\ ${ }^{10}$ Physikalisches Institut, Westfälische Wilhelms-Universität, Wilhelm-Klemm-Strasse, 10, 48149 Münster, Germany \\ ${ }^{11}$ European XFEL, Albert-Einstein-Ring 19, 22761 Hamburg, Germany
}

(Received 29 October 2013; published 12 March 2014)

\begin{abstract}
We report on the dynamics of ultrafast heating in cryogenic hydrogen initiated by a $\lesssim 300 \mathrm{fs}, 92 \mathrm{eV}$ free electron laser $\mathrm{x}$-ray burst. The rise of the $\mathrm{x}$-ray scattering amplitude from a second $\mathrm{x}$-ray pulse probes the transition from dense cryogenic molecular hydrogen to a nearly uncorrelated plasmalike structure, indicating an electron-ion equilibration time of $\sim 0.9 \mathrm{ps}$. The rise time agrees with radiation hydrodynamics simulations based on a conductivity model for partially ionized plasma that is validated by two-temperature density-functional theory.
\end{abstract}

DOI: 10.1103/PhysRevLett.112.105002

PACS numbers: 52.25.Os, 52.20.Fs, 52.27.Gr, 52.50.Jm

Knowledge of thermodynamic properties of matter under extreme conditions is critical for modeling stellar and planetary interiors [1], as well as for inertial confinement fusion (ICF) experiments [2]. Of central importance are the electron-ion collision and equilibration times that determine the microscopic properties of matter related to reflectivity and thermal conductivity. On a macroscopic scale, these affect the depth of mixing layers in Jovian planets [3], as well as the formation of a central hot spot and the assembly of a stable thermonuclear fuel layer in ICF implosions [4-6]. Equilibration times are also crucial for the interpretation of ion heating in recent $Z$-pinch plasma experiments $[7,8]$. Uncertainties in the transport properties in dense matter limit our ability to accurately model such complex systems.

Precise determination of the heat transfer rate from electrons to ions and atoms in dense matter requires the preparation of a uniform sample and sub-pico-second probing. Recent attempts using optical-laser heated thin metal foils are promising, but limited by the nonuniformity of the heated sample and a time resolution of few [9-11].

As a versatile diagnostic tool for warm and dense matter states, x-ray scattering was demonstrated on picosecond [12] and nanosecond time scales [13-15]. With the advent of free electron lasers (FELs) the implementation of volumetric x-ray heating $[16,17]$ and accurate $\mathrm{x}$-ray scattering on the femtosecond time scale is now becoming possible due to the short FEL pulse lengths $(\lesssim 300 \mathrm{fs}$ ), their unprecedented peak brightness, and the high repetition rate [18]. To investigate dynamic processes of warm dense matter on such short time scales, dual-pulse experiments are necessary, where a first pulse generates an excited state that is subsequently probed by a second pulse at well-defined time delays.

Here we have used the split and delay capability [19] of the free-electron laser in Hamburg (FLASH) (as illustrated in Fig. 1) to measure ultrafast heating of dense matter. We produce two pulses of comparable intensity $[20,21]$ to volumetrically heat dense cryogenic hydrogen and subsequently probe it by soft $\mathrm{x}$-ray scattering. The total scattered fraction rises to a peak value of $4 \times 10^{-6}$ within $0.9 \mathrm{ps}$, remaining constant for delays up to $2 \mathrm{ps}$. This dynamics is reproduced with a Saha model for ionization. In contrast, simulations using a quotidian equation of state (QEOS) show quasi-instantaneous heating within the FEL pulse duration of 300 fs.

FLASH was operated at $92 \mathrm{eV}$ soft $\mathrm{x}$-ray radiation to be in a regime where the photon energy is well above the plasma frequency for liquid-density hydrogen $\left(\hbar \omega_{P} \sim 8 \mathrm{eV}\right)$. This leads to a high penetration depth with an absorption length $l_{\mathrm{abs}}=11 \mu \mathrm{m}$ [24], comparable to the hydrogen jet radius of $\sim 9 \mu \mathrm{m}$. The average spectral 


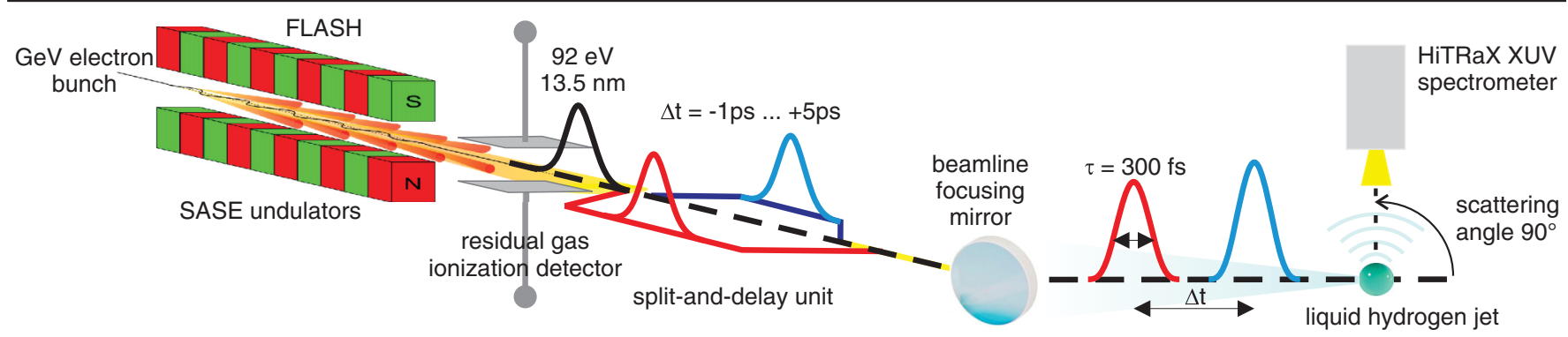

FIG. 1. Schematic of the experimental setup. Horizontally polarized FEL radiation at $92 \mathrm{eV}, 10 \mathrm{~Hz}$ pulse repetition rate, and duration of $\tau \lesssim 300 \mathrm{fs}$ is geometrically divided by a split-and-delay unit [19]. Likewise, a time delay $\Delta t$ between the $\mathrm{X}$-ray pulses is introduced with few-fs precision. The pulses are subsequently focused to a $20 \times 30 \mu \mathrm{m}^{2}$ spot using an ellipsoidal mirror, yielding intensities up to $(27 \pm 0.6) \mathrm{TW} / \mathrm{cm}^{2}$ for the variable-delay branch and $(19 \pm 0.4) \mathrm{TW} / \mathrm{cm}^{2}$ for the fixed branch. The FEL pulses hit a $(9 \pm 2) \mu \mathrm{m}$ radius hydrogen jet with mass density of $0.08 \mathrm{~g} / \mathrm{cm}^{3}$ and temperature of $20 \mathrm{~K}$. Scattering is collected at $90^{\circ}$ relative to the incident FEL radiation in the vertical plane. To discriminate between the scattered XUV photons and plasma self-emission or energetic particles, we employ a variable line space grating spectrograph [22,23].

bandwidth was measured to be $\Delta E / E \approx 1.6 \%$. An upper limit for the FEL pulse duration is estimated from the measured electron bunch duration ( 300 fs FWHM), and individual pulse energies have been recorded by a residual gas ionization detector, yielding an average pulse energy of $(200 \pm 50) \mu \mathrm{J}$. About $20 \%$ of this energy is delivered to the target due to the beam line transmission. The hydrogen jet is extruded from a liquid helium cooled cryostat [25] with $60 \mathrm{~m} / \mathrm{s}$ flow velocity, so that each FLASH pulse scatters from an unperturbed sample. During hydrogen injection the chamber was at a pressure of $10^{-5}$ mbar.

After initial absorption, photoelectrons with kinetic energies of $78 \mathrm{eV}$ impact ionize several atoms on the time scale of the FEL pulse duration [26], leading to an almost homogeneous electron heating throughout the sample. Subsequently, these electrons transfer their heat to the molecules, atoms, and ions. At this photon energy, attenuation via photo absorption has the highest cross section with a small fraction of the incident radiation being also scattered.

$\mathrm{X}$-ray scattering spectra are measured for various time delays up to 5 ps. The number of Rayleigh-scattered photons $N_{\text {scat }}$ per solid angle $\mathrm{d} \Omega$ and per incident photons $N_{\text {inc }}$ of linearly polarized x rays is given by [27]

$$
\frac{1}{N_{\text {inc }}} \frac{\mathrm{d} N_{\text {scat }}}{\mathrm{d} \Omega}=\frac{V n_{i}}{A} Z r_{0}^{2} S(k),
$$

where $V$ is the scattering volume, $n_{i}$ is the heavy particle density (ions and atoms), $A$ is the irradiated area (the focal spot being larger than the sample), $Z=1$ is the atomic number, $r_{0}$ is the classical electron radius, and $S(k)$ is the structure factor for a momentum transfer $k=\left\|\mathbf{k}_{1}-\mathbf{k}_{2}\right\|=$ $0.0348 a_{\mathrm{B}}^{-1}$ (for $90^{\circ}$ scattering angle). Here $\mathbf{k}_{\mathbf{1}}$ and $\mathbf{k}_{\mathbf{2}}$ are the incident and scattered $\mathrm{x}$-ray wave numbers, respectively. Since this value of $k$ is sufficiently close to zero we use the structure factor at zero momentum transfer, $S(k=0)$, which is a measure for the sum of all short- and longrange electron correlations.

For our conditions $k r \ll 1$ and $k D \gg 1$, where $r$ is the mean interparticle separation and $D$ the hydrogen jet diameter. In this regime, scattering probes the collective behavior of a large number of particles.

High-dynamic range spectra at $(90 \pm 2.2)^{\circ}$ scattering angle are measured as a function of time delay by accumulating 300 consecutive exposures at a fixed delay. We employ the HiTRaX spectrometer [22] which has a toroidal mirror $25.5 \mathrm{~cm}$ from the target, providing a large solid angle of $1.9 \times 10^{-3} \mathrm{sr}$. Its spectral dispersion was calibrated in situ using plasma emission lines [23]. Figure 2 shows that between 0 and 1 ps time delay there is a significant increase of the total scattered intensity. We also notice that the spectral shape shows subtle changes of the wings, mostly dominated by the incident FEL spectrum.

To describe the temporal variation of the scattering intensity, we first performed one-dimensional Lagrangian radiation-hydrodynamic simulations with the code HELIOS

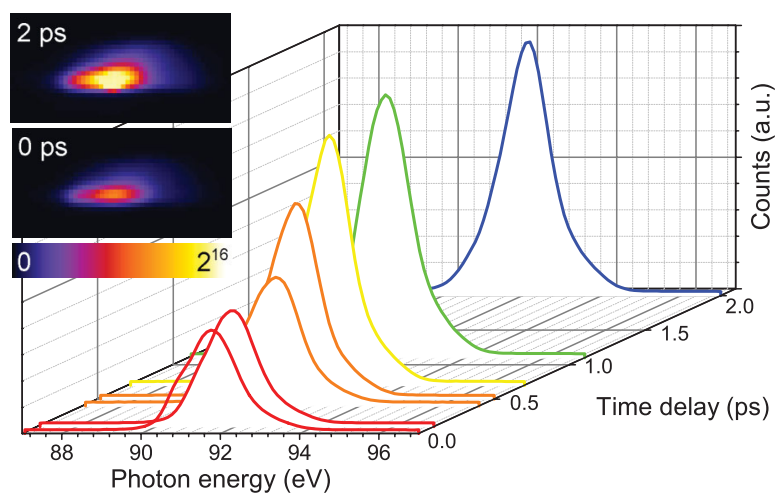

FIG. 2. Experimental scattering spectra as function of time delay. The inset shows two 16-bit raw spectra, each $25 \times 50$ pixels of $(13.5 \mu \mathrm{m})^{2}$ size, with a horizontally oriented spectral axis corresponding to the graph, where the spectral profiles are integrated along the vertical axis. 
[28]. It solves the equations of motion for a fluid considering the electronic and ionic subsystem as comoving, where their energies can be handled separately. The equation of state (EOS) of hydrogen is used from the PROPACEOS package. Both pulses are accounted for in the simulations. The spatially resolved ion density as well as the electron and heavy particle temperatures are extracted from these simulations at the peak of the probe pulse.

The simulation is run with the EOS according to the Saha equation, predicting weak ionization of 4\%-6\% and, consequently, a slow electron-ion energy exchange (of the order of several ps). Within the pump pulse duration, the electronic subsystem is heated to $T_{e} \sim 2 \mathrm{eV}$ (Fig. 3), and energy is subsequently transferred to the heavy particles. Their $T_{i}$ rises from cryogenic temperatures to $\sim 0.25 \mathrm{eV}$ within $1 \mathrm{ps}$ and subsequently continues rising to $0.5 \mathrm{eV}$ at $5 \mathrm{ps}$. Note that in the present case $T_{e}$ and $T_{i}$ do not equilibrate due to the occurrence of the probe pulse.

In order to obtain structural information and determine $S$ $(k=0)$, the plasma conditions derived from HELIOS are taken for each delay time as input parameters for $a b$ initio two-temperature density-functional-theory molecular dynamics (2T-DFT-MD) simulations [29], using the Vienna $a b$ initio simulation package (VASP) [30]. DFT at finite temperatures is implemented in VASP solving the Kohn-Sham equations self-consistently to minimize the free energy of the system as a functional of the local electron density

$$
n(\mathbf{r})=\sum_{i=1}^{N} f\left(\epsilon_{i}, T\right)\left|\phi_{i}(\mathbf{r})\right|^{2},
$$

where the wave functions are weighted with the Fermi distribution $f\left(\epsilon_{i}, T\right)$. The effective potential defining all

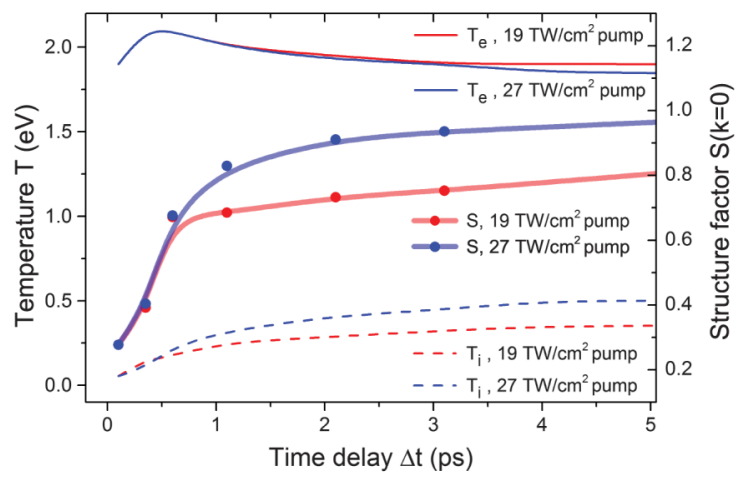

FIG. 3. Simulation of the temporal evolution of the plasma parameters and the structure factor. The temperatures of the electronic and atomic-ionic subsystems are calculated using the 1D radiation-hydrodynamics code HELIOS and a Saha-based EOS. Also shown is the structure factor at $k=0$. No temporal evolution in the initial ion density is observed. The ionization ranges between $4 \%-6 \%$, temporally following the electron temperature dependency. interactions in the system includes ion-background contributions, the Hartree energy, and the exchange-correlation functional. The heavy particle temperature $T_{i}$ is controlled with a Nosé thermostat while the electron temperature $T_{e}$ is defined via the Fermi weighting of the electron distribution. Convergence is checked with respect to the particle number, the $\mathbf{k}$ point sets used for the evaluation of the Brillouin zone, and the energy cutoff for the plane wave basis set. Coulomb interactions between the electrons and ions are treated using projector-augmented wave potentials [31] with a converged energy cutoff of $1400 \mathrm{eV}$. We chose 64 atoms, the Baldereschi mean value point [32], and use the exchange-correlation functional of Perdew, Burke, and Ernzerhof [33], which has been shown to give reasonable results for warm dense matter states [34,35].

In a physical picture, these DFT simulations provide the pair distribution function $g(r)$ of atoms and ions as well as the equation of state data. The structure factor can be written as $S(0)=\kappa_{T} / \kappa_{T}^{\text {id }}$, using the real $\left(\kappa_{T}\right)$ and the ideal $\left(\kappa_{T}^{\text {id }}\right)$ isothermal compressibilities. While the ideal compressibility is simply given by $\kappa_{T}^{\text {id }}=1 /\left(n_{i} k_{B} T\right)$, the real compressibility is obtained from the DFT equation of state via $\kappa_{T}=(\partial \rho / \partial p) / \rho$. The evolution of $S(0)$ in time is shown in Fig. 3. At initial times, we see that $S(0)$ differs from unity indicating a highly correlated system. At later times, $S(0)$ approaches 1 , which can be explained by a transition from cryogenic molecular hydrogen to an almost uncorrelated plasmalike structure with an ideal gaslike compressibility.

Figure 4 shows 2T-DFT-MD simulations of $g(r)$ for different temperatures, which correspond to specific time delays. A pronounced peak in the distribution function is seen at radius $r \sim 0.75 \AA$ that coincides with the distance of the molecular atom pair [34] and characterizes an

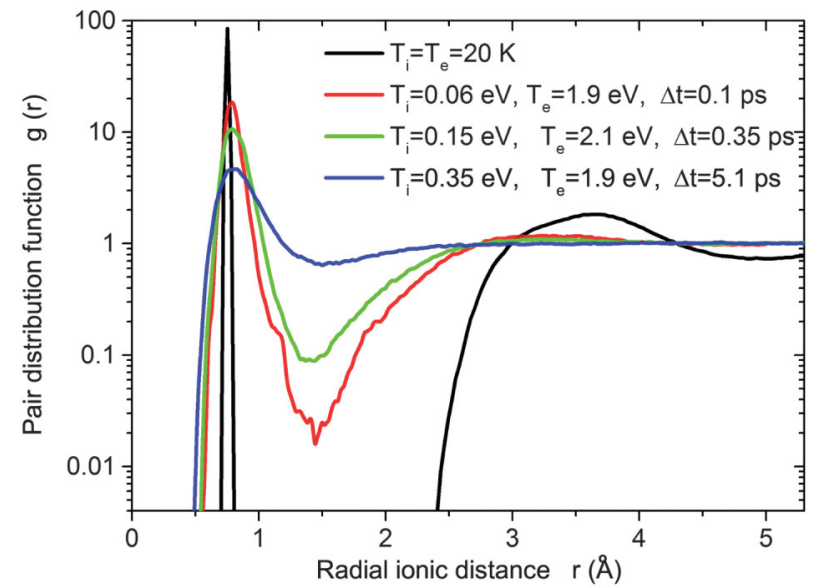

FIG. 4. Shown is the calculated pair distribution function $g(r)$ as a function of the radial interparticle distance $r$ for different time delays and temperatures. For cryogenic hydrogen $(\mathrm{T}=20 \mathrm{~K})$, a peak at $r \sim 0.75 \AA$ indicates the distance in the $\mathrm{H}_{2}$ molecule. With increasing temperature the molecular bonds break and the intensity of the peak drops. 


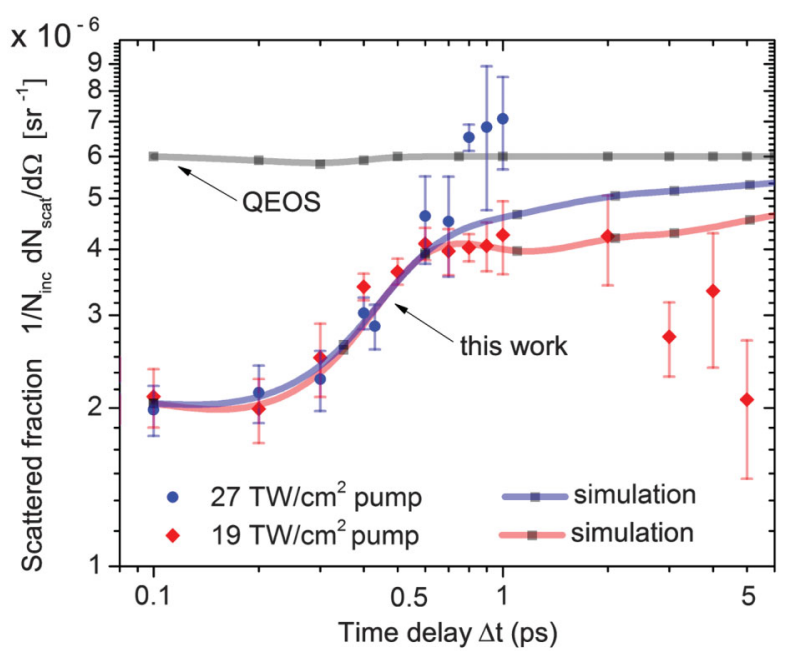

FIG. 5. Temporal evolution of the measured and simulated $\mathrm{x}$-ray scattering. A total of 300 exposures were grouped together by delay, and their errors resemble their root mean square deviations. For the blue data points, ranging from $\Delta t=0 \mathrm{ps}$ to 1 ps only, the more intense $27 \mathrm{TW} / \mathrm{cm}^{2}$ pulse pumps the target, while it is probed by the $19 \mathrm{TW} / \mathrm{cm}^{2}$ pulse. The red data points, ranging from 0 to $5 \mathrm{ps}$, resemble the inverse case where the pump was less intense $\left(19 \mathrm{TW} / \mathrm{cm}^{2}\right)$ than the probe $\left(27 \mathrm{TW} / \mathrm{cm}^{2}\right)$. In both cases, the signal increases within $1 \mathrm{ps}$, and for the more intense pump the amplitude is larger. Simulations based on QEOS (gray) predict no delay dependence for $S(0)$.

undissociated molecular system. With increasing temperature the molecular peak drops and broadens and a continuous distance distribution arises, corresponding to bond breakage and plasma formation.

In Fig. 5 the scattered fraction of the photons is plotted versus time delay. The data were normalized to the incoming photon number $N_{\text {inc }}$ and the XUV spectrometer efficiency $\eta$. The latter quantity is estimated from the specifications of each spectrometer component. Best agreement with the simulations (as shown in Fig. 5) is achieved when multiplying the measured values with a factor of 2.6. In the simulations, the hydrogen jet diameter was estimated to be $18 \mu \mathrm{m}$ from the nozzle diameter and Rayleigh breakup. Assuming a relative error of $\sim 15 \%$ in jet diameter results in 50\% uncertainty in the number of available scatterers.

We observe a significant increase of the signal for time delays $\lesssim 1$ ps. We also performed the experiment with reversed time delay between the x-ray pulses. Stronger pumping of the cryogenic hydrogen (corresponding to $27 \mathrm{TW} / \mathrm{cm}^{2}$ ) leads to more scattering, because a higher pump intensity raises the temperatures further, leading to a less correlated hydrogen system. For the weaker pump $\left(19 \mathrm{TW} / \mathrm{cm}^{2}\right)$, we were also able to measure time delays up to 5 ps. First we observe that after 1 ps the scattering amplitude stops growing and then flattens until about 2 ps. This is due to slower equilibration caused by the smaller temperature difference between electron and heavy particles. The observed slow decrease towards 5 ps could be attributed to fast recombination of ions [36] (requiring a detailed configuration accounting of all excited states) which might be underestimated in our hydrodynamics calculations.

A Gaussian fit to the experimental data yields a valley-topeak time of $(1.1 \pm 0.2)$ ps. After deconvolution of the probe pulse duration, we derive that cryogenic molecular hydrogen responds to an impulsive heating of the electrons within $(0.9 \pm 0.3) \mathrm{ps}$, in good agreement with the temporal evolution based on hydrodynamics simulations.

To cross-check the hydrodynamics results which are based on a conductivity model for partially ionized plasma, we independently extract the time scale of electron-ion collisions from the ab initio DFT simulations by fitting the dynamical conductivity to a Drude relation [37], yielding $\tau_{\text {coll }}=1-3$ fs. This leads to a consistent equilibration time $\tau_{\mathrm{eq}}=m_{p} /\left(2 m_{e}\right) \tau_{\mathrm{coll}}=1-3 \mathrm{ps}$, where $m_{p}$ and $m_{e}$ are the proton and electron mass, respectively. We further prove that a Saha model as implemented in this work is superior to the Thomas-Fermi model as implemented in QEOS [38]. The latter predicts about an order of magnitude too short equilibration times, generating a plasma with $S(0) \sim 1$, well within the FEL pulse duration. Hence, no pump-probe delay dependence for $S(0)$ is predicted using QEOS (see Fig. 5).

Numerical simulations indicate that the Rayleigh-Taylor growth is highly sensitive to thermal conduction [39]. Recently, uncertainties in the heat diffusion coefficient of a factor 10 restrict the prognosis of the mixing layer between compressed DT and beryllium in ICF capsules to a factor $\sim 2$ precision. In the same way, this also puts the predicted depth of mixing layers in Jovian planets into perspective [3,6]. Hence, our results provide important insights and needed experimental data on transport effects, which will shed light on the detailed understanding of dense plasmas.

The authors thank the FLASH machine and experimental team for their great support. The assistance of the Bundesministerium für Bildung und Forschung within the priority research area FSP 301 FLASH, the Deutsche Forschungsgemeinschaft within the SFB 652, CUI, and the VolkswagenStiftung is acknowledged. Partial funding from UK EPSRC Grant No. EP/G007187/1 and from the French Agence Nationale de la Recherche under Grant IRONFEL-ANR-12-PDOC-0011. This work was partially performed under the auspices of the U.S. Department of Energy by Lawrence Livermore National Laboratory under Contract No. DE-AC52-07NA27344 and was supported by LDRD 11-ERD-050. It was partially supported by the DOE Office of Science, Fusion Energy Sciences under FWP 100182. DFT-MD simulations were performed at the NorthGerman Supercomputing Alliance (HLRN). Supporting simulations were performed at the John von NeumannInstitute for computing. 
*Corresponding author.ulf.zastrau@uni-jena.de

[1] N. Nettelmann, B. Holst, A. Kietzmann, M. French, R. Redmer, and D. Blaschke, Astrophys. J. 683, 1217 (2008).

[2] J. Lindl, P. Amendt, R. L. Berger, S. G. Glendinning, S. H. Glenzer, S. W. Haan, R. L. Kauffman, O. L. Landen, and L. J. Suter, Phys. Plasmas 11, 339 (2004).

[3] J. Leconte and G. Chabrier, Astron. Astrophys. 540, A20 (2012).

[4] A. J. Mackinnon et al., Phys. Rev. Lett. 108, 215005 (2012).

[5] S. H. Glenzer et al., Phys. Plasmas 19, 056318 (2012).

[6] S. P. Regan et al., Phys. Plasmas 19, 056307 (2012).

[7] M. G. Haines, P. D. LePell, C. A. Coverdale, B. Jones, C. Deeney, and J. P. Apruzese, Phys. Rev. Lett. 96, 075003 (2006).

[8] E. Kroupp et al., Phys. Rev. Lett. 107, 105001 (2011).

[9] F. Dorchies et al., Phys. Rev. Lett. 107, 245006 (2011).

[10] T. Ao, Y. Ping, K. Widmann, D. F. Price, E. Lee, H. Tam, P. T. Springer, and A. Ng, Phys. Rev. Lett. 96, 055001 (2006).

[11] Z. Lin, L. V. Zhigilei, and V. Celli, Phys. Rev. B 77, 075133 (2008).

[12] A. Kritcher et al., Science 322, 69 (2008).

[13] S. H. Glenzer et al., Phys. Rev. Lett. 98, 065002 (2007).

[14] G. Gregori et al., Phys. Rev. Lett. 101, 045003 (2008).

[15] E. Garcia-Saiz et al., Nat. Phys. 4, 940 (2008).

[16] B. Nagler et al., Nat. Phys. 5, 693 (2009).

[17] S. Vinko et al., Nature (London) 482, 59 (2012).

[18] W. Ackermann et al., Nat. Photonics 1, 336 (2007).

[19] M. Wöstmann, R. Mitzner, T. Noll, S. Roling, B. Siemer, F. Siewert, S. Eppenhoff, F. Wahlert, and H. Zacharias, J. Phys. B 46, 164005 (2013).

[20] M. Bonn, S. Funk, C. Hess, D. N. Denzler, C. Stampfl, M. Scheffler, M. Wolf, and G. Ertl, Science 285, 1042 (1999).

[21] T. Döppner, T. Fennel, T. Diederich, J. Tiggesbäumker, and K. H. Meiwes-Broer, Phys. Rev. Lett. 94, 013401 (2005).

[22] R. R. Fäustlin, U. Zastrau, S. Toleikis, I. Uschmann, E. Förster, and T. Tschentscher, JINST 5, P02004 (2010).
[23] U. Zastrau et al., JINST 6, P10001 (2011).

[24] P. Sperling, R. Thiele, B. Holst, C. Fortmann, S. H. Glenzer, S. Toleikis, Th. Tschentscher, and R. Redmer, High Energy Density Phys. 7, 145 (2011).

[25] S. Toleikis et al., High Energy Density Phys. 6, 15 (2010).

[26] R. R. Fäustlin et al., Phys. Rev. Lett. 104, 125002 (2010).

[27] S. H. Glenzer and R. Redmer, Rev. Mod. Phys. 81, 1625 (2009).

[28] J. MacFarlane, I. Golovkin, and P. Woodruff, J. Quant. Spectrosc. Radiat. Transfer 99, 381 (2006).

[29] Z. Chen, B. Holst, S. E. Kirkwood, V. Sametoglu, M. Reid, Y. Y. Tsui, V. Recoules, and A. Ng, Phys. Rev. Lett. 110, 135001 (2013).

[30] G. Kresse and J. Furthmüller, Phys. Rev. B 54, 11169 (1996).

[31] P. E. Blöchl, Phys. Rev. B 50, 17953 (1994).

[32] A. Baldereschi, Phys. Rev. B 7, 5212 (1973).

[33] J. P. Perdew, K. Burke, and M. Ernzerhof, Phys. Rev. Lett. 77, 3865 (1996).

[34] B. Holst, R. Redmer, and M. P. Desjarlais, Phys. Rev. B 77, 184201 (2008).

[35] W. Lorenzen, B. Holst, and R. Redmer, Phys. Rev. B 82, 195107 (2010).

[36] S. H. Glenzer, W. Rozmus, B. J. MacGowan, K. G. Estabrook, J. D. De Groot, G. B. Zimmerman, H. A. Baldis, J. A. Harte, R. W. Lee, E. A. Williams, and B. G. Wilson, Phys. Rev. Lett. 82, 97 (1999).

[37] K. U. Plagemann, P. Sperling, R. Thiele, M. Desjarlais, C. Fortmann, T. Döppner, H. Lee, S. Glenzer, and R. Redmer, New J. Phys. 14, 055020 (2012).

[38] R. M. More, K. H. Warren, D. A. Young, and G. B. Zimmerman, Phys. Fluids 31, 3059 (1988).

[39] B. Hammel, S. Haan, D. Clark, M. Edwards, S. Langer, M. Marinak, M. Patel, J. Salmonson, and H. Scott, High Energy Density Phys. 6, 171 (2010). 